\title{
A Multi-agent Approach to the Deregulation and Restructuring of Power Industry
}

\author{
Felix Wu, Chris Yeung \\ Department of Electrical \& Electronic Engineering \\ University of Hong Kong \\ ffwu@hkueee.hku.hk \\ Ada Poon \\ Department of Electrical Engineering and Computer Science \\ University of California \\ Berkeley, California \\ Jerome Yen \\ Department of Computer Science \\ University of Hong Kong
}

\begin{abstract}
In recent years, electric utility industry in the whole world has been facing pressure to be deregulated or restructured in order to increase its efficiency, to reduce operational costs or to give consumers more alternatives. The once centralized system planning and operation management must be remodelled to adapt to the new market environment. Subject to the unavoidable constraints, such as, capacity of generation stations, physical limitations of the transmission lines, and demand on days-ahead scheduling, the current trading mechanism needs to be revised so that any party can involve in this freemarket decor. This paper presents a multi-agent approach to resolve the multilateral trading problem. We have implemented a prototype based on Bilateral Shapley Value and Internet technologies. The prototype has been tested with a classical six-bus system.
\end{abstract}

\section{Introduction}

Deregulation and restructuring of utility industry has been an important area for the past decade. Regulatory body, operation environment, technologies, definition of boundary, and the nature of electricity business have changed very fast. Therefore, it is very difficult and almost impossible to use the old rules or regulations, which were designed decades ago even before the creation of computers, to manage and control the current power industry [1, 34]. In the past, production and distribution of electricity had been considered as natural monopoly due to the economic scale [33]. New power plants and transmission systems could cost several billion dollars. Such franchised monopoly, under protection and insulated from competition, obviously had a much weaker incentive in controlling costs and avoiding poor decisions as those competed in open markets. Therefore, customers have been imposed excessive costs which were unfair and unjustifiable [6]. For example, poor decisions in 1970s in expanding power generation capacity by building more nuclear power plants had forced the consumers to pay higher prices to recover the additional costs, such as, the case in Washington state. One of the major motivations to deregulate and restructure the power industry is to avoid the same mistake [36].

As innovations in electrical engineering, material sciences, and supporting information technologies have been developed rapidly, the lowcost production and transmission of electricity have become possible [6]. Therefore, several countries, such as, United Kingdom, New Zealand, Norway, Chile, and Argentina, have taken significant steps toward the deregulation and privatization of their electric power industry $[16,29,33]$.

Generation, transmission, and distribution services used to be bundled together as a single commodity. After restructuring or deregulation, they could be treated as individual service providers and priced their services separately. Therefore, more participants, many of them have been previously excluded in making decision on behalf of themselves, could participate in the trading to create a more cost-effective and more efficient market [7, 27]. Expansion of the power system has been driven by the market demand. Security, reliability, and economic issues have to be separated so that the conflict of interests in the power dispatching can be avoided. This was the major incentive to the development of the Independent System Operator (ISO). How to carry out a smooth transition from the regulated and protected environment to a more 
open and competitive market, ISO may have an important role to play $[1,17]$.

In a restructured market, information structure and decision making process have become more decentralized and more distributed. Therefore, the requirements on the information processing and communication among the participants have also been changed to reflect the changes the market structure. Decentralization of planning, management and operation have created a demand for new methodologies, models and technologies. For example, a workshop had been organized by the joint efforts of Pserc (The Power System Engineering Research Center) and EPRI (The Electric Power Research Institute) to identify the tools and systems in engineering and economics that needed in the future [34].

In such decentralized market, each participant should allow to stay autonomously in a free and open market. In addition, they may be geographically dispersed, Internet and agent technologies can support their communication, negotiation and coordination [3, 20, 24, 35, 37]. Internet and agent technologies can be used together to develop systems to support both planning and operation of utility market [15]. However, the requirements on supporting operation are much higher, because a decision has to be made within seconds if there is a demand for balancing the loading. We will solve the operation problem in our future research. In this paper, we focus on system planning phase.

To prove that Internet and multi-agent systems may be a new alternative to the planning for system expansion, we developed an Internet-based multiagent system to help participants identify better or more appropriate partnerships (or coalitions) within the electricity market, such as, suppliers and consumers. The core of the multi-agent system is a flexible multilateral trading scheme, which was developed based on the Bilateral Shapley Value (BSV) [30, 38].

Market structuring of power industry and a literature review of the deregulation and restructuring of power industry will be discussed in Section 2. The rules for the will be provided in section 3. The agents for the multilateral trading will be discussed in section 3 .

\section{Agents, Cooperative Game Theory and Multilateral Trade, and ISO}

In this section, we will briefly discuss the research in software agents, cooperative game theory, multilateral trading, independent service operator (ISO) and schedule coordinator (SC).

\subsection{Software Agents}

Many applications have been developed recently to remove the burdens of human beings from those tedious and repeating tasks. One of such applications is the software agents. If agents have special capabilities, such as, learning, proactive decision making, and communication, they can be called intelligent agents. Marvin Minsky said [25]:

"My on-line Random House dictionary includes many senses for agent:

When you use the word "agent" in any of these ways, there is no implication that what the agent does is simple. They all suggest the agent is seen as having some specialized purpose. If you need help with making investments, you call a financial agent. If you're looking for a job, you call an employment agent. In the present-day jargon of computer interface agents, the word is used for a system that can serve as a go-between, because of possessing some specialized skills."

The agent approach to support intellectual work, collaboration, information searching and data analysis has created many successful applications and gained significant attention over the past few years. Aparicio has identified eight major application areas that relate to agents [2]. For example, manage systems and networks, manage workflow, and support electronic commerce.

These applications represent a fundamental shift in the human-computer interaction paradigm. As stated in a recent Science article: "...the agent approach transforms the computer user from a worker into a manager [35]. You delegate tasks to a set of agents who do things for you." One of the basic ideas of the agent research is to develop systems which "engage" and "help" different end users to reach their goals [28].

An "agent" can be a program that operates autonomously to accomplish unique tasks without direct human supervision (similar to human counterparts such as real estate agents, travel agents, etc.) [23]. For example, a "spider" who looks for relevant information in the cyberspace[11], an scheduling agent schedules meetings based on constraints or patterns that the agent had learned previously, or an information or e-mail filtering agent who filters news group articles based on the "induced" (or learned) users' profiles [24].

Agents receive assignments and instructions from the owners, then based on the models or knowledge bases, accomplish the assignments. Some agent researchers attempt to address the question: "how should agents interact with each other to form agent teams on the Internet?" Other researchers are more concerned with designing agents who are "robust", “intelligent", and "human-like" [28]. 
Multi-agent systems are special type of agents, which more focus on coordination and communication among agents either to collaboratively accomplish a task [19, 20, 21, 31]. In our multi-agent system, the agents are naturally the generators, customers and coordinators (to be explained later). The objective is thus to derive a workable and profitable coalition partnership under the fair play practice subject to power generation and transmission conditions.

Communication and cooperation are two most important capabilities to the multi-agent systems. Multi-agent systems are designed to have the capability to either, collaborate, for example, decompose a problem and jointly solve the problem, or compete, such as, search for the best deals for the users. The term cooperation used in this paper is assumed to include both collaboration and competition. Communication is vitally important by which relevant information to support cooperation is exchanged. KQML (Knowledge Query and Manipulation Language) is a language that supports the communication among agents [12]. However, agents must do more than just communication. Rational agents must be able to cooperate and negotiate with each other. Design of the communication and negotiation protocols is important. However, so far there is no protocol that dominates this field. One language developed by Barbuceanu and Fox [4] called COOL, which is an extension of KQML. COOL allows the agents to be developed with the capability to make proposals and counter-proposals, accept and reject goals, notify the other agents of goal cancellation or creation.

\subsection{Game Theory and the Multi-Lateral Trading}

Game theory has been an important research area to both mathematics and economics for the past four decades. They can be applied to solve most decision making problems by creating games where the following three conditions are satisfied:

1. There are two or more autonomous decision makers, called players;

2. Each player has a choice of two or more ways of acting, called strategies, such that the outcome of the interaction depends on the strategy choices of all the players;

3. The players have well-defined preferences among the possible outcomes, so that numerical payoffs reflecting these preferences can be assigned to all players for all outcomes.

Application of game theories to solve distributed artificial intelligence (DAI) problems, or more precisely, the coordination and collaboration of agents, is a new but promising research area. We therefore use the multi-agent approach and game theory to model the core of the utility market - the multi-lateral trading mechanism.

Multi-lateral trading mechanism has played an important role in planning and operation of electricity industry. Game theories, especially the cooperative game theories, have been used extensively to identify the trading partners in various trading markets [13, 38]. The purpose of the multilateral trading game is to search for the best coalitions for each player in the game.

However, just searching for the best coalition is not sufficient. How to fairly allocate the costs and the payoffs among the players can be even more important. Among various models, Shapley Value and Bilateral Shapley Value have shown great potential and been used by many researchers [38]

Forming coalition and identify business partners is important in the deregulation and restructuring of power industry $[3,18,32]$. The reason is that the old boundary or regulations have been removed and participants need to find new partners to protect their long-term interests, for example, stable supply of low-price electricity. Many approaches have been used to determine the coalitions or planning for the network expansion, for example, sensitivity analysis [5], Branch-And-Bound Integer Programming [22], Discrete Dynamic Optimization [10], and Least-effort Criterion [26]. However, most of them are centralized approaches and they do not support the decentralized nature in the utility markets after their restructuring or deregulation. In the following, we will briefly discuss the process of forming coalition.

There are four phases of cooperation and coordination among agents in the multi-lateral trading system to form coalition [20]. To simplify the development of the prototype, only bilateral negotiation is considered in which exactly two participants are involved in each discussion or negotiation in forming coalitions.

1. Information Derivation: Each agent submits its local trading requirement such as costs of power generation or profits of selling electricity to an independent service operator (ISO) agent who ensures the feasibility and fairness of trades. It then receives the global information, such as, the average cost of using transmission network.

2. Communication: Every agent exchanges its information with other agents directly or indirectly through a coordinator agent. Each agent continuously gathers information from the ISO concerning the market environment, for example, the network reliability. 
3. Computation: Every agent calculates the total profit and its own share if teaming with other agents. A preference list of other agents will be created in the order of most positive total profits.

4. Bilateral Negotiation: According to the preference list, each agent extends offer to others on the list. At the same time, it may accept another offer which will improves its position and consequently it has to decline the current offer. The process will be iterated until a pre-set duration has arrived and after that, no further negotiation is allowed.

After these four steps, the system would come out with the coalition and the list of players in the coalition. The next phase is the costs or payoffs allocation.

Costs allocation is an important step after formation of coalition. Since each player has contribution to the total payoff of coalition, therefore, it is necessary to go through a process to allocate the costs or profits according to individuals' contributions. During the computation step (Step 3), each agent has to know its own share of profit. There are many solutions to derive the payoff vectors, for example, the core, stable set, and Shapley value etc..

In order to be consistent with how the coalition was formed, we again use the Bilateral Shapley Value (BSV) model [20] to identify the relative weighting of their contributions. For example, consider a network of four agents namely A, B, C and D. The coalition sets are: $\{\{A\},\{B\},\{C\},\{D\}\}$

$\rightarrow\{\{A, B\},\{C, D\}\} \rightarrow\{A, B, C, D\}$. The profit for $\{\mathrm{A}, \mathrm{B}\}$ in $\{\mathrm{A}, \mathrm{B}, \mathrm{C}, \mathrm{D}\}$ will be equal to its $\mathrm{BSV}$, say $P_{A B}$, and the same applies to $\{\mathrm{C}, \mathrm{D}\}$. Then the share of $\mathrm{A}$ in $\{\mathrm{A}, \mathrm{B}\}$ is given by:

$$
\frac{B S V \text { of } A \text { in }\{A, B\}}{B S V \text { of } A \text { in }\{A, B\}+B S V \text { of } B \operatorname{in}\{A, B\}} \cdot P_{A B}
$$

The profits for all the agents are calculated similarly. Besides Bilateral Shapley Value, the concept of Kernel in cooperative game theory can also be used to allocate profit or costs [32].

\subsection{Independent Service Operator and Schedule Coordinator}

In a multilateral trading, the major participants are generators, customers, schedule coordinators and the independent system operator (ISO) [17]. The ISO administers the security and reliability of power generation and transmission, ensures that all constraints and limitations are met. In addition, it releases information to all the market participants, which include hazardous conditions, such as, transmission failure and over-generation.

A schedule coordinator (SC), or simply a coordinator, is responsible for the formation and coordination of a coalition of generators and customers. It promotes multilateral trading which involve two or more players to guarantee that the sum of power generation equals the sum of power consumption plus the losses due to transmission. An SC also has another goals, such as, search for profitable trades and insure that the trades are feasible based on the available information and constraints.

In fact, a SC may as well be a generator, a customer, a broker, or a power exchange (PX) [17], which serves as a daily spot market to balance the supply and demand of electricity with publicly posted prices. A sample dialogue in Figure 1 illustrates a mode of information flow between a coordinator and a participant in which a participant submits a request and the coordinator, after consulting the ISO, replies with an endorsement or a counter proposal if the request cannot be granted.

The ISO, SC, generators, customers, and potential market participants all require intensive communications in order to establish schedules, handle transactions, and perform analysis during the trading process. The trading must be based on the equal opportunity and fair competition principles. As such, each participant should be autonomous and be responsible for own decision making. In this respect, intelligent agents or multi-agent systems are found to be suitable to model such complex, independent and yet cooperative behavior.

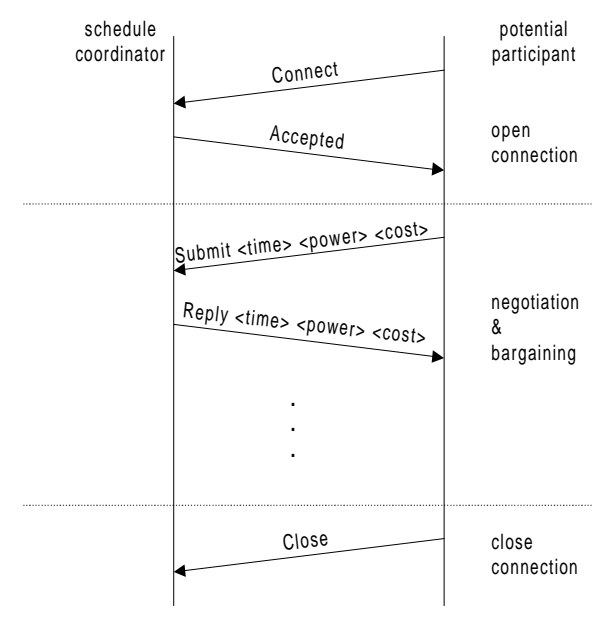

Figure 1. Coordinator dialogue sample

\section{An Example}

In the above section, we have introduced the model and process to form coalitions. In order to illustrate how they work, we use an example in this section. Throughout this section, a simple four-bus system is used to discuss how to model the multilateral trading system. An agent in the game can be a power station (generation) or a group of consumers (consumption). For simplicity, we exclude the cases of fractional power generations or consumption. We 
also assume that any set of power generators or consumption loads attached to the same bus will be represented by the same agent.

In the following, we will examine how the coalition is formed with a simple 4-bus network. Figure 2 depicts a network with bus nodes (1) and (2) represent the generators while nodes (3) and (4) the customers. Other information required is the reactance/p.u. for each transmission line and a transfer admittance matrix (not shown) used in calculating power flow.

\begin{tabular}{|l|l|l|r|}
\hline Line no. & From & To & Reactance / p.u. \\
\hline \hline 1 & $(1)$ & $(2)$ & 0.2 \\
\hline 2 & $(1)$ & $(4)$ & 0.25 \\
\hline 3 & $(2)$ & $(3)$ & 0.25 \\
\hline 4 & $(3)$ & $(4)$ & 0.4 \\
\hline
\end{tabular}

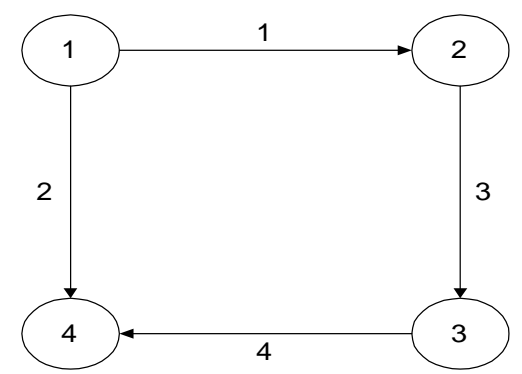

Figure 2. A 4-bus system example

To compute the total profits of a coalition, we also need to consider the transmission cost for every bus line. Here we assume that the cost of transmission varies linearly (Figure 3 ) with its line flow and that the transmission network has infinite capacity.

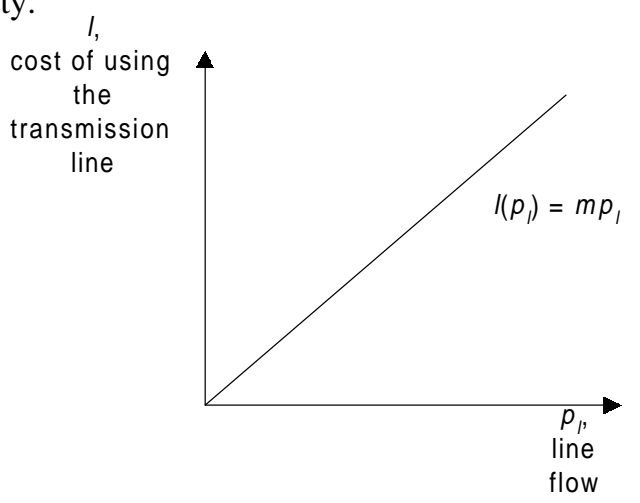

Figure 3. Linear transmission line cost model

Figure 4 displays the global and local information that define the 4-bus system where $m$ in the global table denotes the slope of the above linearity relationship. For the local information, the costs of generation for both generators are the same (i.e. 0.05), as well as the benefits of consumption for the customers (i.e. -0.06).

Global Information:

\begin{tabular}{|l||r|r|r|r|}
\hline Line No. & 1 & 2 & 3 & 4 \\
\hline$m$ & 5 & 5 & 5 & 5 \\
\hline
\end{tabular}

Local Information:

\begin{tabular}{|c||l|l|l|l|}
\hline Bus Node & $(1)$ & 2 & 3 & (4) \\
\hline bus injection & 100 & 100 & 120 & 120 \\
\hline $\begin{array}{c}\text { Cost } \\
\text { generation/ ben } \\
\text { of consumption }\end{array}$ & $\begin{array}{l}0 f .05 \\
\text { fit }\end{array}$ & 0.05 & - & - \\
\hline
\end{tabular}

\section{Figure 4. Global and Local Information}

We formulate the problem as an optimization problem to find the power injection by the entities in a coalition $C, p_{C}$ resulting in optimal profit. The mathematical form of the objective function is given by

$$
\begin{aligned}
& \text { Minimize } \quad u^{T} \cdot C\left(p_{C}\right)+l^{T} \cdot|H \cdot p| \\
& \text { subject to } u^{T} \cdot p_{C}=0 \text {, where } \\
& C=\text { vector of cost / benefit } \\
& \text { functions for agents in } C \\
& p_{C}=\text { vector of power injections by } \\
& \text { agents in } C \\
& l=\text { vector of all transmission } \\
& \text { line costs } \\
& H=\text { transfer admittance matrix of the } \\
& \text { transmission network } \\
& p=\text { vector of all net power injections } \\
& \text { at all buses by agents in } C \\
& u=\text { column vector of all ones }
\end{aligned}
$$

Profit of coalition, therefore is calculated by the following equation,

$$
v(C)=-u^{T} \cdot C\left(p_{C(\min )}\right)-l^{T} \cdot\left|H \cdot p_{(\min )}\right|
$$

Based on the above data and the optimization criteria, the outcome of coalition can be calculated and shown in Figure 5. The difference in the profits for $\{1,3\}$ and $\{1,4\}$ is due to the higher transmission cost induced for $\{1,3\}$ where power needs to flow through both lines 1 and 3 . Also notice that the grand coalition $\{1,2,3,4\}$ naturally results in the global optimum as this is precisely the monopolistic case before deregulation. 


\begin{tabular}{|c|c|c|c|}
\hline Coalition, $C$ & Profit & Power injection /M & $\begin{array}{l}\text { Transmissip } \\
\text { cost }\end{array}$ \\
\hline$\{1\}$ & 0 & & \\
\hline$\{2\}$ & 0 & & \\
\hline$\{3\}$ & 0 & & \\
\hline$\{4\}$ & 0 & & \\
\hline$\{1,2\}$ & 0 & & \\
\hline$\{1,3\}$ & 227 & $\begin{array}{l}p_{1}=45.4545 \\
p_{3}=-45.4545\end{array}$ & 455 \\
\hline$\{1,4\}$ & 368 & $\begin{array}{l}\mathrm{p}_{1}=57.8500 \\
\mathrm{p}_{4}=-57.8500\end{array}$ & 421 \\
\hline$\{2,3\}$ & 368 & $\begin{array}{l}\mathrm{p}_{2}=57.8500 \\
\mathrm{p}_{3}=-57.8500\end{array}$ & 421 \\
\hline$\{2,4\}$ & 227 & $\begin{array}{l}\mathrm{p}_{2}=45.4545 \\
\mathrm{p}_{4}=-45.4545\end{array}$ & 455 \\
\hline$\{3,4\}$ & 0 & & \\
\hline$\{1,2,3\}$ & 515 & $\begin{array}{l}\mathrm{p}_{1}=21.0012 \\
\mathrm{p}_{2}=54.5957 \\
\mathrm{p}_{3}=-75.5969\end{array}$ & 483 \\
\hline$\{1,2,4\}$ & 515 & $\begin{array}{l}\mathrm{p}_{1}=54.5957 \\
\mathrm{p}_{2}=21.0012 \\
\mathrm{p}_{4}=-75.5969\end{array}$ & 483 \\
\hline$\{1,3,4\}$ & 529 & $\begin{array}{l}\mathrm{p}_{1}=80.1359 \\
\mathrm{p}_{3}=-28.6218 \\
\mathrm{p}_{4}=-51.5142\end{array}$ & 544 \\
\hline$\{2,3,4\}$ & 529 & $\begin{array}{l}\mathrm{p}_{2}=80.1359 \\
\mathrm{p}_{3}=-51.5142 \\
\mathrm{p}_{4}=-28.6218\end{array}$ & 544 \\
\hline$\{1,2,3,4\}$ & 1023 & $\begin{array}{l}\mathrm{p}_{1}=68.1821 \\
\mathrm{p}_{2}=68.1821 \\
\mathrm{p}_{3}=-68.1821 \\
\mathrm{p}_{4}=-68.1821\end{array}$ & 682 \\
\hline
\end{tabular}

Figure 5. Coalition Formation of the 4-bus network

\section{Implementation}

In the previous section, we have used a four-bus example to illustrate how to form best coalition and how to allocate profits or costs among the players in the same coalition. In this section, we will discuss how to develop the multi-lateral trading mechanism based on the multi-agent approach to solve the same problems.

There are several systems been developed to help the decision makers in their planning of the expansion or operation of power system, for example, systems that based on Ptolemy or other graphical software [8, 9]. However, they are centralized approaches as mentioned earlier. As mentioned earlier, the power system planning and operation will be decentralized across under the new regulations or new market structures which are geographically dispersed. To meet such challenges, such as, coordination and dissemination of information, we should develop new systems and new methodologies. In the following, the requirements of coordinated multilateral trading, the protocols of communication, the structure of information, and the support for computation will be discussed. These requirements were the foundation for the selection of technologies to develop the target system.

\section{Coordinated Multi-lateral Trading}

The multi-lateral trading model was developed based on two important entities: the Independent System Operator (ISO) and the Schedule Coordinator (SC). ISO controls the transmission dispatching to ensure the security and reliability of power transmission. Only when security is threatened, ISO would intervene to reduce or adjust the schedules submitted by the SC. SC is the one who, based on economic analysis, proposes coalitions of generators and consumers. It also arranges multi-lateral tradings to make sure that the sum of power generation equals or more than the sum of consumption plus the losses due to transmission.

\section{Protocols and Infrastructure of Communication}

During trading periods or planning stage, ISO, SC, generators and consumers, and other potential participants require extensive communication to set up schedule, handle transactions and conduct analysis. The protocols and infrastructure at this moment still wide open for research. In order to provide high-quality and stable communication and to use the existing communication infrastructure, we selected applications, such as the World-wide Web (WWW), and protocols, TCP/IP, that run over the current Internet.

\section{Structure of Information}

On the electricity market, ISO has to release information about loading condition of power transmission to SC. Based on the information, SC arranges trades and forward the messages back to ISO. In such case, ISO is a server who receives the requests from the $\mathrm{SC}$ and other potential market participants responds accordingly through the protocol as discussed above. The SC is also a server who receives requests from the potential trading partners to monitor the request queues. Therefore, the whole system is best to be modelled with clientserver architecture. To support the back-end database management, we selected the mSQL to provide fast access to the stored data.

\section{Support Computation}

ISO ensures security and reliability of power transmission, maintains real-time load balance, and guarantees that constraints are met. All these operations are computation intensive. In order to reduce the turnaround time of computation, we had 
to design our system so that it supports distributed processing. In the real-world cases, an ISO distributes processing loads to several processors, which can be heterogeneous and geographically dispersed. There are many approaches and applications to solve the computation problem which encountered in such distributed environment. In order to save the time and efforts in the development of our system, we selected existing software tools to perform the computations, such as, optimization.

The multi-agent based multi-lateral trading mechanism is designed to allow decision makers to explore more alternatives in planning for new expansion or searching for new partners. Based on the above requirements, it was developed with the following technologies:

\section{Java}

Java is an object-oriented and distributed programming language. It was specifically designed to develop systems that run on the Internet. Its network centricity, sandbox security model, and platform independence make it an ideal candidate for the development of agent-based tools. In particular, the object-oriented (OO) nature of Java enables the modelling of generic agents. Any specific agent can be modelled based on the inheritance characteristic as existed in class.

\section{JATLite}

Our multi-agent system is developed based on JATLite which provides a set of lightweight Java packages for the development of multi-agent systems. JATLite, developed at the Stanford University, supports several layers of agent communication through message passing. Knowledge Query and Manipulation Language (KQML) is one of these layers that we have been used in the system.

\section{Ptolemy, Tcl/Tk, MATLAB®, and mSQL}

While the multi-agent model being more or less a front-end application, the entire system indeed includes a host of integrated tool set for back-end support and processing. Ptolemy offers a flexible framework supporting heterogeneous system specification, simulation, and design. Each model of computation is a domain, which consists of an extensible library of functional blocks. We have employed a distributed optimization tool built with Ptolemy to solve the transmission constrained economic dispatch problem in power system analysis.

MATLAB has been used to compute the optimal profit for a participant in forming coalition which can be formulated as a linear programming problem. $\mathbf{m S Q L}$ is a distributed lightweight database engine providing fast access to relational data organization. All power line and network characteristics are captured in the database. Finally, Tcl/Tk supplies the glue logic to link the above modules dynamically and can even be integrated with the Java/Internet environment.

As part of the system, we have also implemented an prototype of the ISO, from which some of the less sensitive information can be disseminated to all the participants through the Internet.

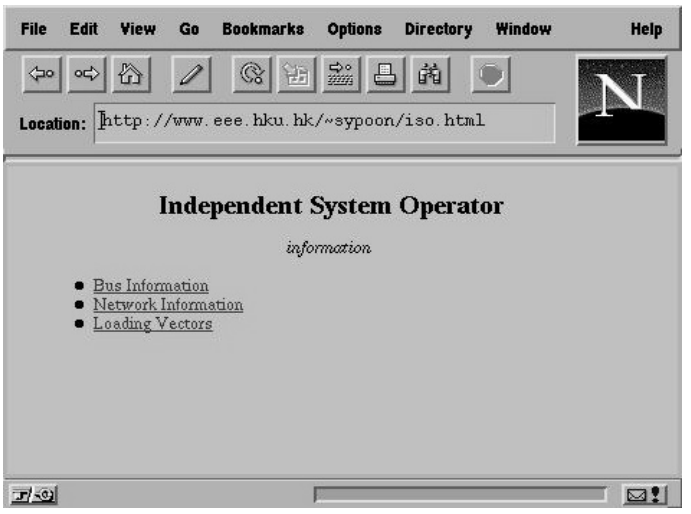

Figure 6. ISO web page

Figure 6 shows a simple ISO web page which allows any participant to query a particular power line network, electrical specification of generators and loads, and the loading vector of transmission lines.

Figures 7 and 8 tabulate the detail transmission line topology and power characteristics of a 6-bus sample taken from [34]. All the data are resided in the ISO database.

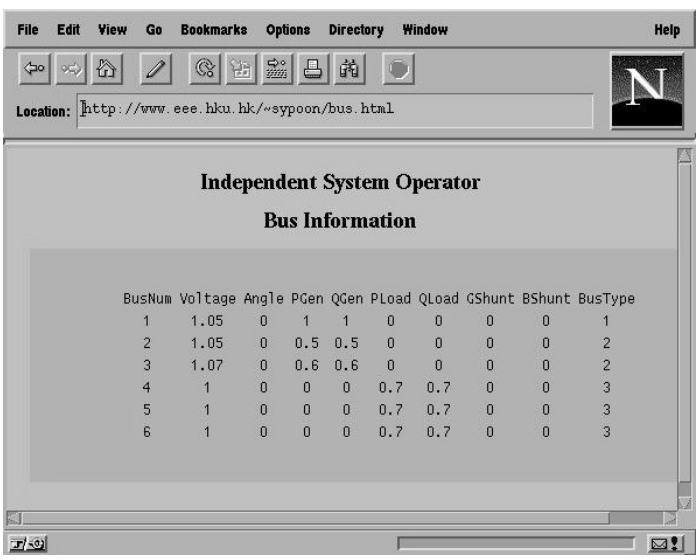

Figure 7. Bus information web page 


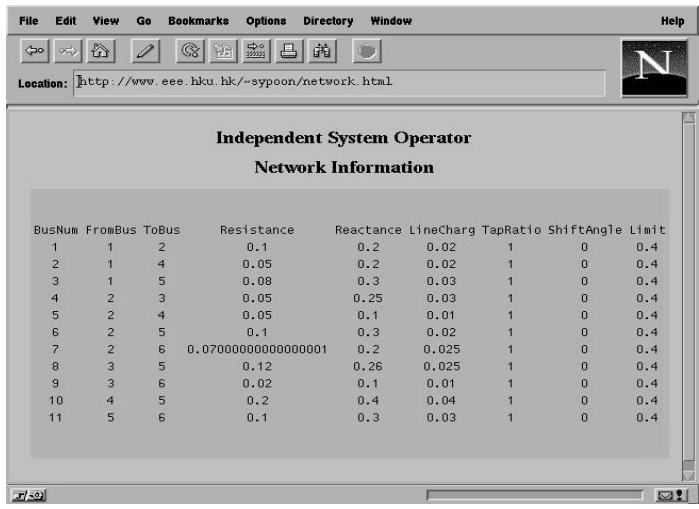

Figure 8. Network information web page

For simplicity there are only two SCs representing all the six generators and customers. The SCs establish trade with the potential partners through the simple dialogue as shown in Figure 1. After bargaining and negotiation with the potential partners, the SCs developed preferred schedules resided in the databases of the two SCs.

The ISO collects schedules from both SCs during each time period and load them into the database. Before the next operating day, the ISO sums the generation and consumption on each bus for each time period. All the transfer of information are done through the Internet and among different agents. The ISO then check the feasibility of all the schedules. To check if trades are feasible, the ISO calculates and solve the network of flow problem based on the information in the database. If there is a overloading in transmission, the ISO initiates the congestion management protocols to relieve the congestion. In such case, the ISO has to reduce the generation and consumption as requested by the participants. Such process can be a repeated process until overloading is no longer exists.

Such process is running in the backend, therefore, it is necessary to create an user interface for the users to monitor the progress and the activities among the agents. The interface was developed with Java and JATLite so that it can be accessed through Internet. The agent server (Figure 9) is responsible for disseminating global information from database, bookkeeping the registered customers, obtaining coalition status from other agents, and terminating the negotiation cycle.

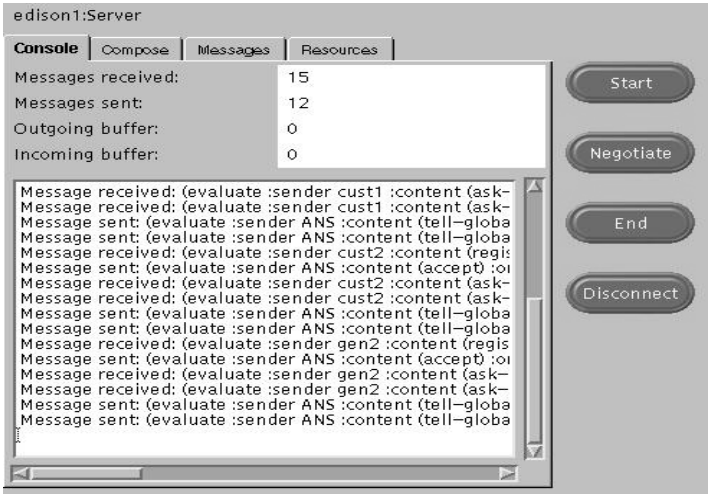

Figure 9. A server agent's panel

Figure 10 shows the process of one agent requested to join the market. On figure 10 , it is easy to see that the message was sent from the agent to the ISO. ISO received the request, processed it and sent the acceptance message back to the agent.

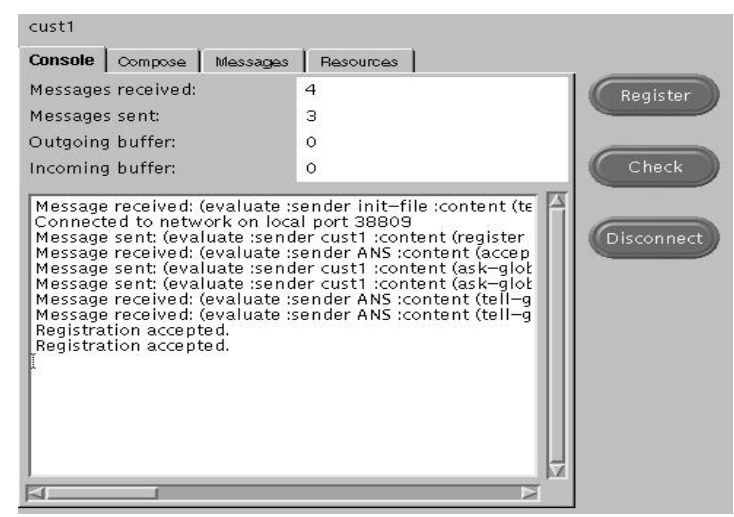

Figure 10. A customer agent's panel

Figures 11 and 12 shows the panels of bus nodes (2) and (3) respectively after negotiation among the agents. From both panels, users can monitor or trace the preference lists, the profits that calculated based on the information the agent received from other agents, and the decision or message that sent to the other agents.

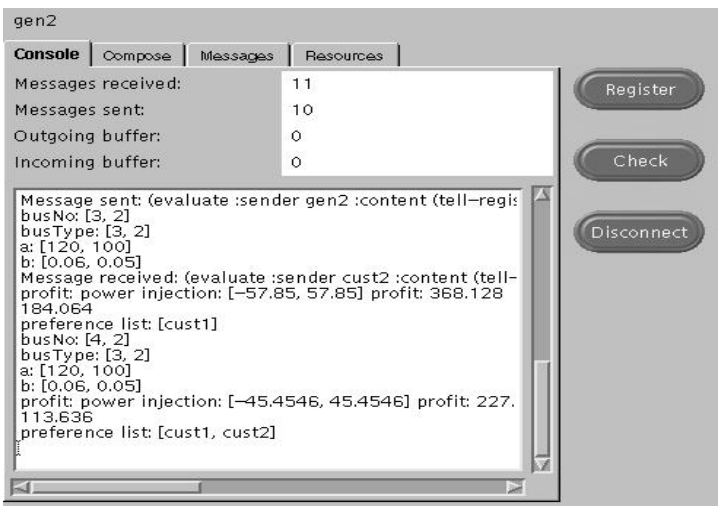

Fig. 11 Bus Node (2) AFTER negotiation 


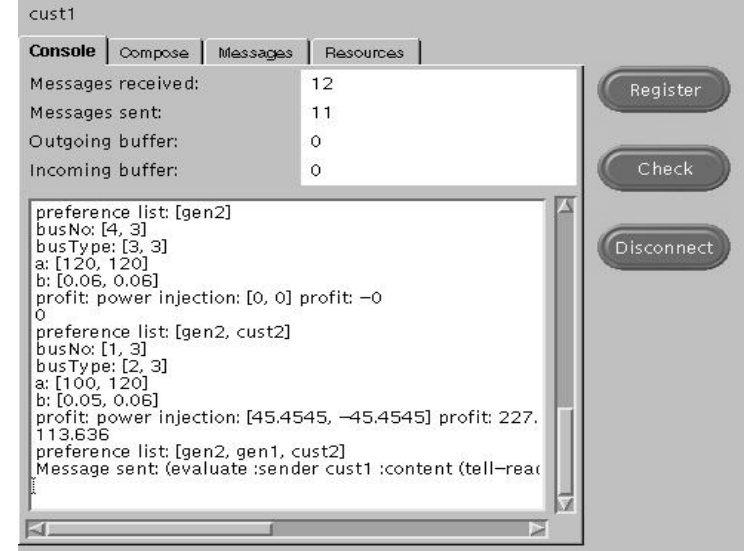

Fig. 12 Bus node (3) AFTER negotiation

\section{Conclusion}

In this paper, we have presented a multi-agent based approach to assist the planning and operation of utility market. Such approach is fully centralized and that is closer to the markets after deregulation or restructuring. This approach can be used not only to support the operation, but also as a simulation system for the users to explore different alternatives in their planning stage.

The major benefits of using such approach are the following:

1. Decision makers can participate in modelling the behavior of the agents, for example, costs, goals, and preferences, and let the system searches the best partners. Compare with a centralized approach, each agent has higher freedom in designing its own strategies or negotiation rules. Normally the centralized approach just assume that all the agents or agents who belong to the same category, such as, generators, use the same set of rules.

2. It allows the users to explore different alternatives within short time. For example, if one agent has captured the strategies of the other agents, then the agent can generate different scenarios to identify the best strategy for bargaining or negotiation.

The implementation has considered Internet or Intranet connectivity by using Java as the core development tool. Therefore, our system can over the barriers that come from distance and also the users do not need to invest on acquiring dedicated communication lines.

\section{REFERENCES:}

[1] Alvarado, F. L., Camfield, R. J., and Rajaraman, R., "Open Transmission Access: An Efficient Minimal
Role for the ISO", in Proceedings of HICSS-30, pp. 571-580, 1997.

[2] Aparicio, G., "The Role of Intelligent Agents in the Information Infrastructure", IBM, USA, 1995. http://activist.gpl.ibm.com:81/WhitePaper/ptc2.htm.

[3] Aumann, R.J., and Myerson, R., "Endogenous Formation Of Links Between Players And Of Coalitions: An Application Of The Shapley Value", in "The Shapley Value: essays in honour of Lloyd S. Shapley," R.B., Roth, A.E., Chapter 12 pp. 175-278, Cambridge University Press, Cambridge, England, 1988.

[4] Barbuceanu, M. and Fox, M. X., Cool: A Language for Describing Coordination in Multi-Agent Systems. In Proceedings of the First International Conference on Multi-Agent Systems (ICMAS-95), pp. 17-24, San Franscisco.

[5] Bennon, R.J., Juves, J.A., and Melioppoulos, A.P., Use of sensitivity analysis Automated Transmission Planning, IEEE Transactions on Power Apparatus and Systems, vol. PAS-101, n.1, 1982.

[6] Bushnell, J., and Stoft, S., Transmission and Generation Investment in a Competitive Electric Power Industry, PWP-030, May 1995.

[7] Chao, H., and Peck, S., A Market Mechanism For Electric Power Transmission, Journal of Regulatory Economics: 10:25-59, 1996.

[8] Contreras, J., Losi, A., Russo, M., and Wu. F.F., Distopt, a Tool for Modeling and Performance Evaluation of distributed Optimization Environments, submitted to IEEE PES '96 SM.

[9] Contreras, J., Losi, A., Russo, M., and Wu, F.F., An application of DistOpt to Power System Optimization Problems, submitted to IEEE PES '96 SM.

[10] Dusonchet, Y.P., and El-Abiad, A.H., Transmission Planning Using Discrete Dynamic Optimization, IEEE Transactions on Power apparatus and Systems, vol. PAS-89, 1973.

[11] Etzioni, O and Weld, D., "A Softbot-based Interface to the Internet", Communications of ACM, Vol. 37, No. 7, pp. 72-79, July 1994.

[12] Finin, T., Fritzson, R., McKay, D., and McEntire, R., "KQML as an Agent Communication Language", in Proceedings of the Third International Conference on Information and Knowledge Management CIKM'94, ACM Press, November 1994.

[13] Gately, D., Sharing The Gains From Regional Cooperation: A Game Theoretic Application to Planning Investment in Electric Power, International Economic Review, vol. 15, n.1, February, 1974

[14] Harsanyi, J., Rational Behavior And Bargaining Equilibrium In Games And Social Sciences, Cambridge University Press, NY, 1977.

[15] Hermans, B., "Intelligent Software Agents on the Internet”, Tilberg University, The Netherlands, 1996.

[16] Hunt, S. and Shuttleworth, G., "Unlocking the Grid", IEEE Spectrum, July 1996

[17] "Independent System Operator [ISO] and Power Exchange [PX] Communications Infrastructure Systems and Services Design and Acquisition Project Request for Proposal [RFP]", August 231996.

[18] Kahan, K. P. and Rapoport, P., Theories of Coalition Formation, Lawrence Erlbaum Associates, Publishers, Hillsdale, NJ, 1984. 
[19] Ketchpel, S.P., Coalition Formation Among Autonomous Agents, Proceedings of MAAMAW-93.

[20] Klusch, M. and Shehory, O., "Coalition Formation Among Rational Information Agents", appeared in Artificial Intelligence 1038 Subseries of Lecture Notes in Computer Science: Agents Breaking Away, Springer Verlag, pp. 204-217, 1996.

[21] Kuwabara, K., Ishida, T., and Osato, N.: "AgenTalk: Describing Multiagent Coordination Protocols with Inheritance", Proc. 7th IEEE International Conference on Tools with Artificial Intelligence (ICTAI '95), p.460-p.465 (1995).

[22] Lee, S.T.Y., Hocks, K.L., and Hnyilicza, E., Transmission Expansion Of Branch-And-Bound Integer Programming With Optimal Cost-Capacity Curves, IEEE transactions on Power Apparatus and Systems, vol. PAS-93, n.5, 1974

[23] Maes, P., "The Dynamics of Action Selection". In Proceedings of IJCAI-89, pp. 991-997, 1989.

[24] Maes, P., "Agents that Reduce Work and Information Overload", Communications of ACM, Vol. 37, No. 7, pp. 30-40, July 1994.

[25] Minsky, M., "A Conversation with Marvin Minsky about Agents", Communications of ACM, Vol. 37, No. 7, pp. 23-29, July 1994

[26] Monticelli, A., Santos Jr., A., Pereira, M.V.F., Cunha, S.H., Parker, B.J., and Praca, J.C.G., Interactive Transmission Network Planning Using a Least-Effort Criterion, IEEE Transactions on Power apparatus And Systems, vol. PAS-101, n.10, October 1982.

[27] Murphy, L. and Wu, F. F., "An Open Design Approach for Distributed Energy Management Systems", IEEE Transaction on Power Systems, vol. 8, no. 3, pp. 1172-1179, August 1995.

[28] Riecken, D., "Intelligent Agents", Communications of ACM, Vol. 37, No. 7, pp. 18-21, July 1994.

[29] Rudnick, H., "Pioneering electricity reform in South America", IEEE Spectrum, August 1996

[30] Shapley, L.S., The value of an n-person game, Contributions to the Theory of Games, in H.W. Kuhn and A.W. Tucker, eds., Princeton University Press, 1953

[31] Shehory, O., and Kraus, S., Coalition formation among autonomous agents: strategies and complexity, Lecture Notes in Artificial Intelligence No. 957, From reaction to Cognition, pp. 57-72, C. Castelfranchi and J.P. Muller (eds.), Springer-Verlag, Berlin, 1993.

[32] Shehory, O., and Kraus, S., A kernel-oriented model for coalition formation in general environments: Implementation and results, Proceedings AAAI-96, Portland, OR, 1996

[33] Tabors, R., "Lessons from the UK and Norway", IEEE Spectrum, August 1996.

[34] Thomas R. J. and Schneider, T. R., "Underlying Technical Issues in Electricity Deregulation", Proceedings of the HICSS-30, pp. 561-570, 1997.

[35] Waldrop, M. M., "Software Agents Prepare to Sift the Riches of Cyberspace", Science, Vol. 265, pp. 882-883, August 1994.

[36] Wood, A. J. and Wollenberg, B. F., "Power Generation, Operation and Control”, John Wiley and Sons, 1996.
[37] Woodridge, M. J. and Jennings, N. R., "Agent Theories, Architectures, and Languages: A Survey". In M. J. Woodridge and N. R. Jennings, editors, Intelligent Agents. Springer-Verlag, Berlin, 1995.

[38] Wu, F. F. and Varaiya, F., "Coordinated Multilateral Trades for Electric Power Networks: Theory and Implementation", Program on Workable Energy Regulation PWP-031, June 1995. 